\title{
Effektive Schmerzlinderung beim Rezidiv eines Rektumkarzinoms mittels Radiofrequenztherapie
}

Bei der perkutanen Radiofrequenztherapie (RF-Therapie) wird eine lokale Gewebedestruktion durch Koagulationsnekrose erreicht. Dabei wird die elektromagnetische Energie durch Ionenbewegung in Wärme umgewandelt. Es gibt bereits umfassende Untersuchungen über die RF-Therapie am Tiermodell (Rossi et al., Tumori 1990; 76: $54-57$ ). Sie wird derzeit in verschiedenen Bereichen als eine Alternative zur chirurgischen Intervention untersucht. Größere Studien zur RF-Therapie von primären und sekundären Lebertumoren liegen bereits vor (Goldberg et al., Am J Radiol 1998; 170: 1023 -1028). Die Wirkung der RF-Therapie zeigte in posttherapeutischen CT- bzw. MRT-Kontrollen eine mehr als $80 \%$ ige Tumornekrose bei ca. 84\% der behandelten sekundären Lebertumoren (Solbiati et al., Radiol 1997; 202: 195 -203). Eine weitere Anwendung ist die Ablation von Osteoidosteomen (Rosenthal et al., J Bone Joint Surg 1998; 80: 815-821). Ein interessanter Therapieansatz liegt in der lokalen Ablation bei der Trigeminusneuralgie zur neurologische Schmerzlinderung als Alternative zur medikamentösen Therapie und Alkoholinjektion (Onofrio, J Neurosurg 1975; 42: 132 -139). Bei 121 von 135 Patienten wurde eine effektive Analgesie erreicht. Wir möchten in dem vorliegenden Fall eine Möglichkeit der effektiven Schmerzlinderung bei einem ausgedehnten Rezidiv eines Rektumkarzinoms vorstellen.

\section{Fallbeschreibung}

Es stellte sich ein 56jähriger Patient in deutlich reduziertem Allgemeinzustand mit starken ischialgiformen Schmerzen vor.

Vor 4 Jahren wurde ein Rektumkarzinom diagnostiziert und mit einer neoadjuvanten Radiochemotherapie und anschließender anteriorer Rektumextirpation $\left(\mathrm{pT}_{3} \mathrm{pN}_{2} \mathrm{MOG}_{2}\right.$ ) behandelt. Bei einem nach knapp 3 Jahren aufgetretenen präsakralen Rezidiv wurde eine abdominoperineale Rektumextirpation mit endständigem Sigmoidstoma und eine Resektion des Os coccygeum sowie des Os sacrum $S_{2}-S_{5}$ durchgeführt und bei $R_{1}$ Resektion eine Radiatio angeschlossen.

Im Rahmen der Tumornachsorge wurde nach 14 Monaten ein 2. Lokalrezidiv im Präsakralraum rechts entdeckt. Die Hauptbeschwerden des Patienten zu dem Zeitpunkt waren starke Schmerzen im Steißbereich, die hauptsächlich in das rechte Bein ausstrahlten. Diese wurden durch eine in der MRT dargestellte Infiltration des Plexus lumbosacralis rechts, aus dem der N. ischiadicus hervorgeht, verursacht. Eine Schmerzlinderung war auch unter hochdosierter Morphiumgabe mit $180 \mathrm{mg}$ Morphin täglich nicht zufriedenstellend. Subjektiv hat der Patient den stark verminderten Wachheitsgrad als erheblich belastend betrachtet. Alternativ zur Morphiumpumpe wurde die transdermale Applikation ohne ausreichende Schmerzlinderung abgebrochen; einen periduralen Katheter lehnte der Patient ab. Der Patient wurde uns zur Schmerzlinderung mittels RF-Therapie vorgestellt. In einem ausführlichen Aufklärungsgespräch wurde der Patient über die möglichen Nebenwirkungen derTherapie informiert. Neben Infektion und Blutungen könnten ferner durch Schädigung des Plexus lumbosacralis auch motorische und sensible Ausfälle im Versorgungsgebiet auftreten. Der Patient wurde auch über das Risiko einer Anästhesia dolorosa aufgeklärt. Das Risiko bei der RF-Ablation bei Trigeminusneuralgien beträgt ca. $2 \%$ (Onofrio, J Neurosurg 1975; 42: 132 - 139). Aufgrund des starken Leidensdruckes des Patienten wurde das mögliche Auftreten motorischer Ausfälle einschließlich Blasen-Mastdarmstörungen akzeptiert.

Unter CT-Sicht wurde zunächst eine Einfachelektrode mit ihrer Spitze mediolateral des vermuteten Verlaufs des $\mathrm{N}$. ischiadicus in die Tumormasse eingeführt (Abb.1) und die Erdung auf dem Oberschenkel platziert. Als Zweites wurde eine Dreifachelektrode in gleicher Sitzung mit der Absicht einer Tumorvolumenreduktion im Tumor platziert. Die freie, nicht isolierte Elektrodenspitze hatte eine Länge von $3 \mathrm{~cm}$, das Kaliber

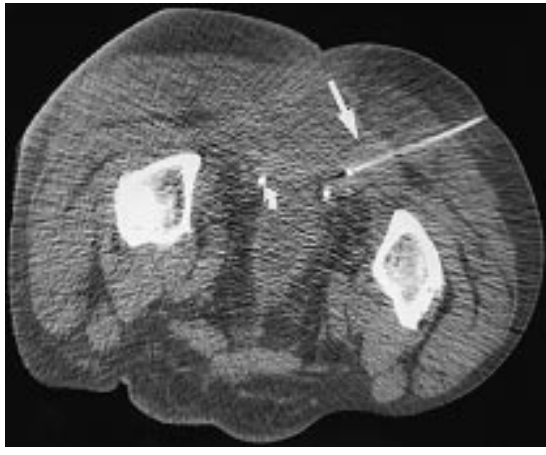

Abb.1 Darstellung der Einfach-Radiofrequenzelektrode mit Lokalisation des nicht isolierten Elektrodenendes im Tumorsubstrat (gerader Pfeil), welches den rechten N. ischiadicus in Höhe des ehemaligen Foramen ischiadicum majus infiltriert. Der gebogene Pfeil markiert den kontrastierten Harnleiter. Man beachte die fehlende Darstellung des Os coccygeum bei Zustand nach Resektion.

der Elektrode betrug 18 Gauge. Die angewendete Leistung der jeweiligen Elektrode lag bei 140 bzw. $200 \mathrm{~W}$ und wurde durch Impedanzmessung während der RF-Applikation überwacht. Die Therapiedauer betrug jeweils $12 \mathrm{~min}$. Direkt im Anschluss an die Sitzung wurde eine Kontroll-CT der Region durchgeführt, um die induzierte Nekrose zu dokumentieren und eine mögliche Blutung auszuschließen (Abb. 2). Eine längerfristige Verlaufskontrolle mittels einer CT wurde für einen Zeitpunkt in ca. 3 Monaten vereinbart. Bei kurzfristig auftretender erneuter Beschwerdesymptomatik konnte eine Wiederholung der RF-Therapie auch früher erfolgen.

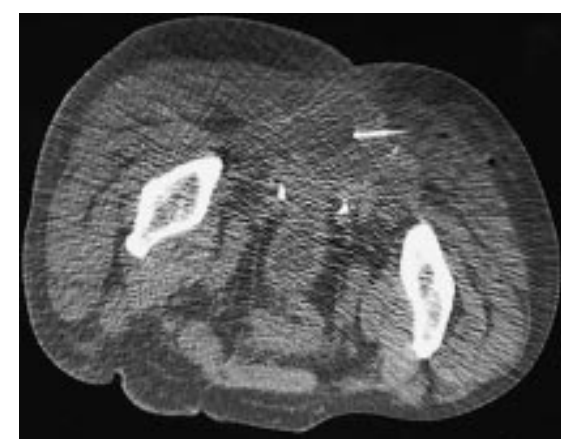

Abb.2 Das Kontroll-CT direkt im Anschluss an die RF-Applikation zeigt die ausgedehnte Koagulationsnekrose in dem Turmorrezidiv am ehemaligen Foramen ischiadicum majus rechts.

Der Patient hat die RF-Therapie komplikationslos vertragen. Es traten keine Nebenwirkungen auf. Die Schmerzthe- 
rapie konnte für einen Zeitraum von etwa 6 Wochen reduziert werden.

\section{Diskussion}

Mit der RF-Therapie können durch Überwärmung des Gewebes auf $>50^{\circ} \mathrm{C}$ lokalisierte Gewebenekrosen erzeugt werden. Im vorgestellten Fall wurde eine mittelfristige Schmerzfreiheit des Patienten erzielt, die durch eine medikamentöse Therapie nicht erreicht werden konnte. Des Weiteren wurde durch Ablation von Tumorgewebe in der gleichen Sitzung eine größere Nekrose erzeugt, die zu einer möglichen Entlastung des umgebenden gesunden Gewebes durch Dekompression führte. Der Vorteil der RF-Therapie liegt unter anderem darin, dass die induzierte Gewebenekrose unabhängig von der Art des Weichteilgewebes (Tumor- oder gesundes Gewebe) ist und somit die Anwendung nicht auf bestimmte Organe oder Tumorarten beschränkt ist. Das Ziel der Behandlung im vorgestellten Fall lag in der reinen Palliation vor allem der die Lebensqualität stark einschränkenden Schmerzen bei fehlenden anderen Therapiemöglichkeiten und nicht in einer Tumorpalliation. Dieser Fall zeigt eine weitere Anwendungsmöglichkeit der Radiofrequenztherapie auf, wenn konventionelle Therapieoptionen nicht mehr vertretbar erscheinen.

E. Hein, P. Rogalla, Berlin 